\title{
Determinación de parámetros operativos para la producción de extracto de saponinas de Cucumis dipsaceus "jaboncillo de campo"
}

\section{Determination of operative parameters for production of saponins extract of Cucumis dipsaceus "hedgehog gourd"}

Segundo Seijas Velásquez ${ }^{1}$, (iD Priscilla Alexandra Seijas Bernabé ${ }^{\natural}$, (D) Nadia Stefanía Seijas Bernabé$^{2}$, (DD Ludwig Emmanuel Salgado Rodríguez ${ }^{3}$ y (D) Marco Antonio Alva Castañeda ${ }^{3}$

${ }^{1}$ Universidad Nacional de Trujillo. Trujillo, Perú
${ }^{2}$ Universidad Privada Cesar Vallejo. Lima, Perú
${ }^{3}$ Instituto de Investigaciones en Ciencia y Tecnología. Lima, Perú

Recibido: 01/03/2021

Revisado: 26/03/2021

Aceptado: 30/03/2021

Publicado: 31/ 06 / 2021

\section{RESUMEN}

Cucumis dipsaceus "jaboncillo de campo", es una especie vegetal de la familia de las cucurbitáceas considerada como maleza en nuestra región, cuyo fruto presenta metabolitos secundarios de importancia industrial y farmacológica destacando entre ellos los flavonoides, glicósidos, alcaloides, taninos y saponinas. Este último compuesto posee propiedades tales como surfactante, espumante, antiinflamatorio y hepatoprotector. Debido a las propiedades que presenta las saponinas se propuso el presente estudio cuyo objetivo principal fue determinar los parámetros operativos: temperatura, tiempo y relación de sólido/solvente en el proceso de producción de extracto de saponina a partir del fruto de Cucumis dipsaceus "jaboncillo de campo". El estudio se inició con un pretratamiento de los frutos el cual consistía en las operaciones de limpieza, pulpeado y secado. Los experimentos se efectuaron mediante ensayos extractivos a nivel de laboratorio para determinar los parámetros óptimos de operación. Luego se procedió al diseño de los equipos que intervienen en el proceso: destilación al vacío, filtrado y secado, así como se obtuvo un programa computarizado en Visual Basic 10 con la finalidad de proceder al escalamiento piloto respectivo. Para determinar la presencia y concentración de las saponinas en el producto final, se realizó los ensayos de hemólisis de glóbulos rojos y prueba de la espuma o afrosimétrica. Se obtuvo un rendimiento de $73,5 \%$ en la producción de saponinas con los respectivos parámetros operativos: de relación sólido/solvente 1, temperatura 600C y tiempo de extracción 30 minutos. Palabras clave: Saponina, parámetros operativos, extracto, optimización, escalamiento.

\section{ABSTRACT}

Cucumis dipsaceus " hedgehog gourd ", is a plant species of the family of cucurbits considered as weeds in our region, whose fruit has secondary metabolites of industrial and pharma- 
cological importance highlighting among them the flavonoids, glycosides, alkaloids, tannins and saponins. The latter compound has properties such as surfactant, foaming agent, anti-inflammatory agent and hepatoprotection. Due to the properties of saponins, the present study was proposed whose main objective was to determine the operating parameters: temperature, time and solid/solvent ratio in the production process of saponin extract from the fruit of Cucumis dipsaceus " hedgehog gourd ". The study began with a pre-treatment of the fruits which consisted of cleaning, pulping and drying operations. The experiments were carried out through extractive laboratory-level tests to determine the optimal operating parameters. Then the design of the equipment involved in the process was carried out: vacuum distillation, filtering and drying, as well as a computer program in Visual Basic 10 was obtained in order to proceed to the respective pilot scaling. To determine the presence and concentration of saponins in the final product, assays of red blood cell hemolysis and foam or afrosymmetric test were performed. A yield of $73,5 \%$ was obtained in the production of saponins using the operating parameters: solid / solvent ratio 1, temperature $600 \mathrm{C}$ and extraction time 30 minutes. Keyswords: Saponin, operating parameters, extract, optimization, scaling.

\section{INTRODUCCIÓN}

Las actividades antropogénicas de diversa índole: económica, tecnológica, sociales entre otras traen una serie de consecuencias negativas a diversos ecosistemas presentándose esta situación durante décadas y este aumento se presenta a medida en que la sociedad interactúe con mayor frecuencia con el medio ambiente, ya que estas interacciones son más complejas a medida que pasa el tiempo (Güçlü-Üstündag,y Mazza, 2007). A pesar de que hay situaciones excepcionales que puedan disminuir estas interacciones, como es el caso de la emergencia global sanitaria dada actualmente por la presencia de la enfermedad pandémica denominada COVID-19 producida por el virus SARS-CoV-2, en donde existen una serie de medidas socio-sanitarias tomadas por diversos países para disminuir las tasas de contagio, y donde se ha observado una disminución de la contaminación en diversos ambientes; pero que al retomar las actividades ya sean graduales o en su totalidad volverá el desequilibrio ecológico (Lolli et al.,2020).
Cabe resaltar que la degradación ecológica no solo produce la disminución y/ o extinción de diversas especies en la flora y fauna sino también causan una serie de problemas de salud en el ser humano (Güçlü-Üstündag,y Mazza, 2007; Villacrés et al., 2019). Uno de los ecosistemas donde hay un mayor daño es en los sistemas acuáticos, debido a la diversidad y cantidad de efluentes que son vertidos en diversos cuerpos de agua entre los que se encuentran los detergentes domésticos considerándose como contaminantes de naturaleza orgánica, los cuales suelen ser muy perjudiciales ya que pueden desencadenar el proceso biológico denominado eutrofización ; por la presencia de fosfatos que algunos detergentes puedan presentar, es por ello que la comisión europea legisló desde el 2012 acerca de la concentración de los fosfatos en la elaboración de detergentes proponiendo la sustitución por fosfonatos y policarboxilatos. Los resultados aún no son concluyentes acerca de si estos compuestos causan algún efecto en los cuerpos de agua porque pueden causar un 
menor porcentaje de eutrofización pero faltaría determinar el nivel de biodegradación en el agua (Ahumada et al.,2016; Prado,2018). Y otro compuesto presente en los detergentes que produce un serio impacto ambiental, son los surfactantes o tensioactivos, los cuales se encuentran en concentraciones relativamente elevadas en la composición de los detergentes ya que son los agentes activos que se encargan de la solubilización de los lípidos y las proteínas que se encuentran en los elementos que requieren ser limpiados o higienizados, debido a esa acción es que causan un daño a nivel celular pues desestabilizan a la membrana celular la cual es de naturaleza lipídica, por lo que son afectados una serie de organismos acuáticos; por lo que se está realizando estudios con la finalidad de proponer agentes tensoactivos que posean una alta tasa de velocidad de biodegradación en condiciones aeróbicas (Bruneton, 2001 ; Hernández, 2006).

Actualmente se tiene conocimiento que la velocidad de degradación de los surfactantes es lenta. Es por ello que en búsqueda de nuevos principios activos que cumplan con los requerimientos de baja ecotoxicidad y una alta tasa de biodegradación se avizoran a las saponinas como una alternativa a la producción de detergentes ecológicos (Francis et al., 2002).A las saponinas se les ha reportado que poseen actividades biológicas semejantes al dodecillbencensulfonato de sodio, el cual es uno de los principios activos que posee la mayoría de los detergentes siendo las saponinas una alternativa adecuada puesto que son biodegradables ecológicos (Francis et al., 2002).

Estos metabolitos vegetales se les han determinado una variedad de propiedades de importancia en el área industrial, farmacológica y biomédica. En el aspecto industrial se pue- den emplear en la fabricación de diversos materiales como los ladrillos acústicos, placas, films y papeles fotográficos y cerámica. Por su capacidad de formar espuma se les emplea en la elaboración de extinguidores de incendios del tipo de espuma y pastas dentífricas, así como para la elaboración de champús, jabones líquidos y preparados cosméticos mientras que en la industria alimentaria también la emplean para la formación de espuma en bebidas tal como refrescos y cervezas (Prado,2018).

Debido a que se reportó que las saponinas presentan actividad analgésica y antiinflamatoria, son empleadas para la elaboración de pomadas de frotación muscular, además poseen efecto mucolítico porque provocan un aclaramiento del mucus denso, dado a esa acción pueden emplearse para la producción de expectorantes (Gianna, 2013; Rijai,2019) .Entre otras actividades biológicas, Bruneton (2001) determinó que las saponinas presentan actividad hemolítica y antilipémica está ultima se debe a su capacidad de disminuir los niveles de colesterol LDL en suero (Ahumada et al., 2016).

Según su estructura química, se les consideran heterósidos (glucósidos) muy frecuentes en los vegetales, se caracterizan por ser de carácter no reductor y que por procesos de hidrólisis ácida o enzimática producen uno o más azúcares y un componente no azucarado llamado aglicona o genina (Gianna,2013;Rijai,2019). Los azúcares más frecuentes constituyentes de los dos tipos de saponósidos son la glucosa, arabinosa, ramnosa, galactosa y xilosa, y en los saponósidos triterpénicos también es frecuente el ácido glucurónico (Gianna,2013; Prado ,2018).

Estos metabolitos secundarios pueden ser clasificados como: saponinas esteroidales y saponinas triterpénicas, estructuralmente 
se diferencian en que las primeras han eliminado 3 de sus radicalesmetilo mientras que las triterpénicas los mantienen intactos. Además, Hernández (2006) menciona que algunos triterpenos no presentan el sistema policíclico característico del esterano (Bruneton, 2001; Hernández y Hermosilla, 2014).

También se emplea subclasificaciones, entre ellas está por el número de posiciones de sustitución de los glúcidos en la estructura de la aglicona o genina, por lo que pueden ser monodesmosídicos (el azúcar o moléculas glicósidicas se unen por una única posición a la genina) y bidesmosídicos (la molécula glicosídica se une por dos puntos a la genina. Los de carácter monodesmosídico se dan con preferencia en raíces, cortezas y semillas, mientras que los bidesmosídicos, se presentan con mayor frecuencia en los tejidos de asimilación que se encuentran conformando parte de las hojas y las ramas tiernas además de presentar mayor grado de hidrosolubilidad en contraste con los monodesmosídicos (Bruneton, 2001; Hernández y Hermosilla, 2014).

Por otro lado, el contenido de saponinas en las plantas depende de diversos factores sean exógenos como tales como, el tipo de cultivo y localización geográfica o de tipo endógenos como la edad de la planta, estado fisiológico, la o el órgano vegetal (Flores,2013).

Debido a la diversidad de usos en donde se podrían emplear las saponinas se están realizando diversos estudios para determinar métodos para una óptima producción teniendo en cuenta el proceso de extracción principalmente con alcoholes ya que son solubles en estas sustancias.

Con respecto a la identificación preliminar de las saponinas, los métodos empleados se basan en las siguientes características: 1) producción de espuma al ser agitadas sus soluciones acuosas; 2) producción de hemólisis eritrocítica; 3) toxicidad en animales poiquilotérmicos, principalmente en los peces (se denominan sapotoxinas), le produce parálisis de las agallas; y 4) presencia de una reacción positiva en la prueba de Liebermann-Burchard. En el caso que sean saponinas esteroidales reaccionan manifestando colores que van desde el azul y viran hasta el verde mientras que las saponinas triterpénicas las tonalidades van desde la coloración rosa, rojo hasta pueden virar al violeta (Flores, 2013).

En relación a las especies investigadas acerca de este principio activo, existe una gran diversidad de especies vegetales que se ha reportado la presencia de saponinas entre ellas está Cucumis dipsaceus llamado "jaboncillo de campo" "jaboncillo de monte" o pepino diablito, familia Cucurbitaceae. Su nombre común es pepino erizo es una especie originaria de Arabia y África tropical y adventicia en América (Flores, 2013; Gianna et al,2012). Botánicamente, se le describe como una hierba trepadora, con presencia de flores amarillas, los frutos están provistos de valvas y de coloración amarillenta, cubiertos de pelos de forma de espinas, generalmente se desarrolla en toda la costa peruana, en suelos salitrosos, húmedos, campos de cultivo, acequias, campos abonados y cercos ubicados entre los 0 a 1000 m.s.n.m. en los departamentos de La Libertad, Lambayeque, Ancash, Cuzco, Lima, Piura, Cajamarca y Piura. (Mostacero et al,2002).

La importancia de obtener saponinas en esta especie es de gran importancia puesto que se le brindaría un valor industrial a una planta considerada 
invasora en los cultivos y muy desagradable por sus pelillos urticantes (Gianna et al, 2012).

Los estudios que se han realizado con esta especie son mayoritariamente a nivel de laboratorio, por lo que la presente investigación propone producir saponinas a partir del fruto de $\mathbf{C u c u - ~}$ mis dipsaceus "jaboncillo de campo" a escala piloto optimizando el proceso de extracción.

\section{MATERIAL Y MÉTODOS}

\section{Obtención de Materia Prima}

Material Biológico: Se colectó las plantas de la especie Cucumis dipsaceus." Jaboncillo de campo" en el distrito de Simbal . Los ejemplares se procedieron a realizar su respectiva limpieza y separar sus frutos, luego se procedió a realizar el secado en un secador solar por 72 horas.

Implementación del módulo de extracción de saponinas

Se implementó el módulo del sistema de extracción cuyo diseño y dimensionamiento se basaron previos estudios preliminares a escala laboratorio. Este sistema estuvo con formado por una autoclave con chaqueta de vapor y dispositivo para control de temperatura, para evaluar tres factores: Relación solido a extractante (alcohol de $70^{\circ}$ ), Temperatura y tiempo de extracción.

\section{Proceso de extracción de saponinas a partir} de los frutos de C. dipsaceus

El diseño experimental en el estudio de este proceso fue de tipo factorial, modelo $3 \mathrm{k}$ ya que presentó tres factores y tres niveles (taba 1 y 2 ), empleando el Diseño Placket \& Burman (P\&B).

Tabla 1. Factores y dominio experimental

\begin{tabular}{|c|c|c|c|}
\hline \multirow[t]{2}{*}{ Factores } & \multicolumn{3}{|c|}{ Dominio Experimental } \\
\hline & $\operatorname{Nivel}(0)$ & $\operatorname{Nivel}(1)$ & Nivel (2) \\
\hline$X_{1}$ : Relación sólido/extractante & $1 / 4$ & $1 / 2$ & 1 \\
\hline$X_{2}:$ Temperatura de extracción $\left(C^{\circ}\right)$ & 50 & 60 & 70 \\
\hline$X_{3}$ : Tiempo de extracción (min.) & 10 & 20 & 30 \\
\hline
\end{tabular}

Determinación de los parámetros operativos en el proceso de extracción etanólica de saponinas

En la extracción se empleó etanol de $70^{\circ} \mathrm{C}$ se procedió a realizar la respectiva maceración por 48 horas a temperatura ambiente con la finalidad de ablandar los tejidos celulares. Posteriormente se procedió a decantar y realizar el proceso de extracción tomando en cuenta los parámetros de relación sólido / extractante; temperatura y tiempo de extracción para cada uno de los tratamientos en el sistema de extracción (tabla 2). 
Tabla 2. Matriz de experimentos de un diseño factorial completo $3^{3}$ y plan de experimentación

\begin{tabular}{|c|c|c|c|c|c|c|c|}
\hline & Matri & xperime & & Plan de experim & Itación & & Respuesta \\
\hline Grupo & $\mathrm{X}_{1}$ & $x_{2}$ & $x 3$ & $\begin{array}{c}\text { Relación } \\
\text { sólida/extractante }\end{array}$ & $\begin{array}{c}\text { Temperatura de } \\
\text { extracción } \\
\text { (C) }\end{array}$ & $\begin{array}{l}\text { Tiempo de } \\
\text { extracción } \\
\text { (minutos) }\end{array}$ & $\begin{array}{c}\text { Concentración } \\
\text { de saponinas } \\
\text { en muestra } \\
(\mathrm{mg} / \mathrm{g})\end{array}$ \\
\hline S1 & 0 & 0 & 0 & $1 / 4$ & 50 & 10 & $\mathrm{Y}_{1}$ \\
\hline \$2 & 0 & 0 & 1 & $1 / 4$ & 50 & 20 & Y2 \\
\hline \$3 & 0 & 0 & 2 & $1 / 4$ & 50 & 30 & Y3 \\
\hline S4 & 1 & 0 & 0 & $1 / 2$ & 50 & 10 & $\mathrm{Y}_{4}$ \\
\hline S5 & 1 & 0 & 1 & $1 / 2$ & 50 & 20 & Y5 \\
\hline S6 & 1 & 0 & 2 & $1 / 2$ & 50 & 30 & Y6 \\
\hline S7 & 2 & 0 & 0 & 1 & 50 & 10 & 17 \\
\hline$\$ 8$ & 2 & 0 & 1 & 1 & 50 & 20 & Y8 \\
\hline s9 & 2 & 0 & 2 & 1 & 50 & 30 & Y9 \\
\hline$\$ 10$ & 0 & 1 & 0 & $1 / 4$ & 60 & 10 & $\mathrm{Y}_{10}$ \\
\hline S11 & 0 & 1 & 1 & $1 / 4$ & 60 & 20 & Y11 \\
\hline$\$ 12$ & 0 & 1 & 2 & $1 / 4$ & 60 & 30 & $\mathrm{Y} 12$ \\
\hline $\mathrm{S} 13$ & 1 & 1 & 0 & $1 / 2$ & 60 & 10 & $\mathrm{Y} 13$ \\
\hline S14 & 1 & 1 & 1 & $1 / 2$ & 60 & 20 & Y14 \\
\hline $\mathrm{S} 15$ & 1 & 1 & 2 & $1 / 2$ & 60 & 30 & Y15 \\
\hline S16 & 2 & 1 & 0 & 1 & 60 & 10 & Y16 \\
\hline \$17 & 2 & 1 & 1 & 1 & 60 & 20 & Y17 \\
\hline S18 & 2 & 1 & 2 & 1 & 60 & 30 & Y18 \\
\hline \$19 & 0 & 2 & 0 & $1 / 4$ & 70 & 10 & Y19 \\
\hline$\$ 20$ & 0 & 2 & 1 & $1 / 4$ & 70 & 20 & $Y_{20}$ \\
\hline S21 & 0 & 2 & 2 & $1 / 4$ & 70 & 30 & Y21 \\
\hline $\mathrm{S} 22$ & 1 & 2 & 0 & $1 / 2$ & 70 & 10 & Y22 \\
\hline$\$ 23$ & 1 & 2 & 1 & $1 / 2$ & 70 & 20 & Y23 \\
\hline$\$ 24$ & 1 & 2 & 2 & $1 / 2$ & 70 & 30 & Y24 \\
\hline$\$ 25$ & 2 & 2 & 0 & 1 & 70 & 10 & Y25 \\
\hline S26 & 2 & 2 & 1 & 1 & 70 & 20 & Y26 \\
\hline$\$ 27$ & 2 & 2 & 2 & 1 & 70 & 30 & Y27 \\
\hline
\end{tabular}

\section{Determinación de Saponinas}

Con la finalidad de determinar la presencia y concentración de las saponinas en el producto obtenido, se propuso realizar los ensayos de la espuma y hemólisis de glóbulos rojos.

Ensayo de Hemólisis:Está prueba se empleó para determinar cualitativamente la presencia de saponinas. Se acondicionó 3 sistemas compuestos por suspensión de glóbulos rojos humanos en solución salina diluida, y se añadió una solución de las muestras obtenidas de cada uno de los tratamientos para adicionar a los tubos de ensayos con los sistemas de glóbulos rojos. Si los glóbulos rojos se rompen (lisan o hemolizan), se asume que la prueba es positiva (presencia de saponinas).
Ensayo de la Espuma: Esta es una prueba denominado prueba de Koziol o afrosimétrica y es para determinar preliminarmente la concentración de saponinas en el extracto.

Para lo cual, se pesó $0,5 \mathrm{~g}$ de muestra (concentrado) de cada uno de los 27 grupos experimentales y se procedió a colocarlos en un tubo de ensayo (sistemas), después se le añadió 5,0 $\mathrm{mL}$ de agua destilada y se tapó el tubo. Se agitó el tubo por un periodo de 30 segundos y se dejó en reposo por 30 minutos, después otra vez se agitó por 20 segundos.

Se repite la operación de dejar en reposo durante 30 minutos más, para luego realizar la agitación por 30 segundos y finalmente se co- 
locaron los sistemas en reposo por un tiempo Luego para calcular el porcentaje de saponinas de 5 minutos y terminado este periodo se reali- por muestra se empleó la siguiente ecuación, zó la medición respectiva de la altura de la es- obtenida a partir de la utilización del estándar puma formada en cada uno de los 27 sistemas. o patrón de saponina (Figura 1 y tabla 3 ).

Tabla 3. Diferentes concentraciones del estándar de saponina y la respectiva altura de espuma obtenida en los ensayos

\begin{tabular}{cc}
\hline \multicolumn{2}{c}{ Estándar de saponina } \\
\hline Concentración (mg/L) & $\begin{array}{c}\text { Altura de espuma } \\
\text { (cm) }\end{array}$ \\
\hline 240 & 2,86 \\
360 & 3,89 \\
480 & 5,14 \\
600 & 6,51 \\
720 & 7,49 \\
840 & 8,59 \\
\hline
\end{tabular}

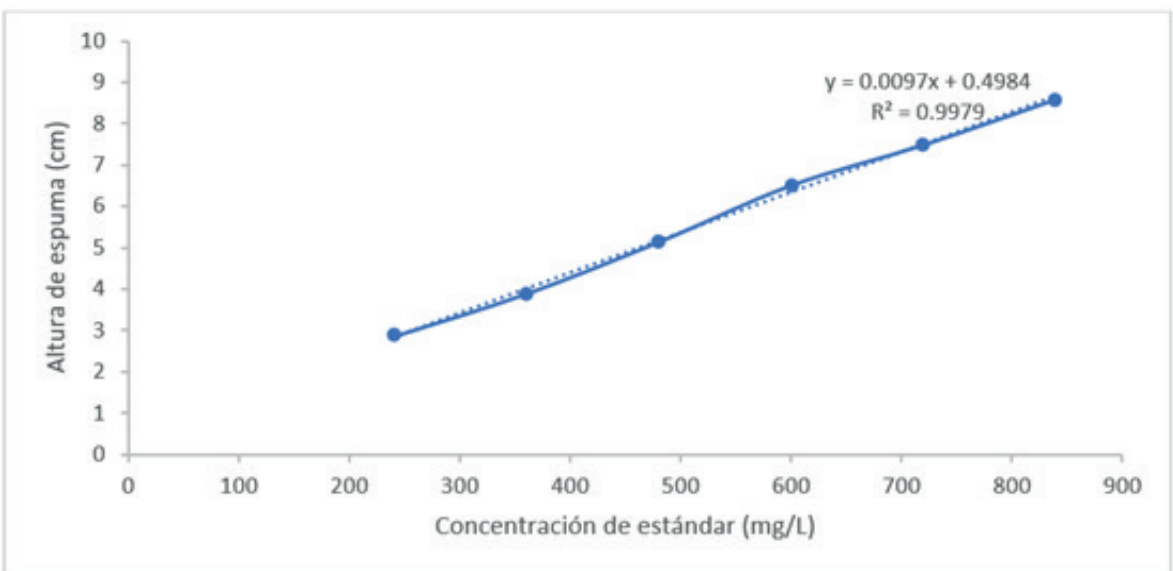

Figura 1. Altura de la espuma según la concentración de saponinas de la muestra patrón. 
La anterior ecuación es para obtener la concentración de saponinas en $5 \mathrm{~mL}$ de la muestra de extracto: (x): $y=0,0097 x+0,4984$.

Mientras que para calcular el porcentaje de saponinas de la muestra se empleó la siguiente ecuación:

$\mathrm{Z}=\mathrm{X} / \mathrm{W} * 100$

Dónde:

$\mathrm{X}=\mathrm{mg}$ de saponina en $5 \mathrm{ml}$ de solución acuosa obtenido de ecuación (1) $\mathrm{Y}=$ Altura de espuma en cm (de los 27 tratamientos) $\mathrm{Z}=$ Porcentaje de saponina en el extracto $\mathrm{W}=\mathrm{mg}$ de extracto en $5 \mathrm{ml}$ de solución acuosa.

\section{RESULTADOS Y DISCUSIÓN}

Según los resultados obtenidos (tabla 4), el tratamiento S18 cuyos parámetros operativos fueron Relación de materia prima /extractante $1: 1$, temperatura de extracción $60^{\circ} \mathrm{C} \mathrm{y}$ tiempo de extracción: 30 minutos; se obtuvo una mayor concentración de saponinas con un $73,5 \%$ en extracto de Cucumis dipsaceus.
Tabla 4. Porcentaje de saponinas en extracto de fruto de Cucumis dipsaceus y prueba cualitativa de presencia de hemólisis según grupo experimental

\begin{tabular}{|c|c|c|}
\hline $\begin{array}{c}\text { Grupo } \\
\text { experimental }\end{array}$ & $\begin{array}{c}\% \% \text { de } \\
\text { saponinas en } \\
\text { extracto }\end{array}$ & $\begin{array}{c}\text { Presencia de } \\
\text { Hemólisis }\end{array}$ \\
\hline S1 & 43,5 & + \\
\hline S2 & 46,5 & + \\
\hline $\mathrm{S} 2$ & 49,8 & + \\
\hline S4 & 52,1 & + \\
\hline S5 & 55,8 & + \\
\hline S6 & 58,5 & + \\
\hline S7 & 53,7 & + \\
\hline S8 & 57,9 & + \\
\hline S9 & 59,3 & + \\
\hline S10 & 55,6 & + \\
\hline S11 & 58,9 & + \\
\hline S12 & 61,6 & + \\
\hline S13 & 62,4 & + \\
\hline S14 & 67,3 & + \\
\hline S15 & 70,9 & + \\
\hline S16 & 65,6 & + \\
\hline S17 & 70,3 & + \\
\hline S18 & 73,5 & + \\
\hline S19 & 57,2 & + \\
\hline $\mathrm{S} 20$ & 58,1 & + \\
\hline S21 & 59,7 & + \\
\hline $\mathrm{S} 22$ & 57,8 & + \\
\hline S23 & 59,5 & + \\
\hline S24 & 61,2 & + \\
\hline S25 & 58.5 & + \\
\hline S26 & 62,6 & + \\
\hline S27 & 64,8 & + \\
\hline
\end{tabular}


Contrastando con lo obtenido por Alarcón (2016) que obtuvo un $67 \%$ de saponinas en extracto de Sapindus saponaria "choloque", empleando como extractante n-butanol mientras que se reportó que el extracto obtenido del fruto de Sapindus rarak se obtuvo una óptima extracción empleando solo metanol en contraste con soluciones de agua/acetona y agua/ metanol a diferentes proporciones (3/1;1/1 y 1/3) para ambos tipos de soluciones, siendo el de menor eficiencia las soluciones que emplearon acetona; la temperatura para todas estas operaciones fue de $50^{\circ} \mathrm{C}$ (Castellanos et al., 2011); en el caso de la presente investigación se obtuvo mayor porcentaje empleando como extractante al etanol al $70^{\circ} \mathrm{C}$ a una temperatura de $60^{\circ} \mathrm{C}$, ya que a temperaturas mayores puede presentarse una degradación de las saponinas (Bajad et al., 2019).

La naturaleza anfifílica (o anfipática) de las saponinas permite la extracción con agua o soluciones hidroalcohólicas empleando diferentes técnicas, cabe resaltar que el tipo de extractante va a depender de la solubilidad que posean estos principios activos, en el caso de ser saponinas triterpenoides como los que posee $C$. dipsaceus son más solubles en alcohol (Castellanos et al., 2011; Bajad et al., 2019).

Diversos estudios abocados al proceso de extracción reportan que generalmente los mejores solventes para las saponinas son el butanol y el metanol en diversas investigaciones en Chenopodium quinoa, Sapindus saponaria, Sapindus rarak, semillas de Momordica cochinchinensis (Paredes y Solar, 2007;Castellanos et al., 2011 ; Flores, 2013; Bajad et al., 2019).En el caso de Dodonaea viscosa el solvente con el que se obtiene un mayor poder extractivo es con solución de etanol al 20\% (Bajad et al., 2019).
Pero se debe tener en cuenta que si estos principios activos serán empleados en la industria alimentaria y principalmente farmacológica el proceso de extracción a escala industrial debe emplearse componentes inocuos en las operaciones implicadas ya que pueden quedar trazas y por ende volverse tóxicas, por ello es la importancia de estudiar no solo el tipo de solvente a emplear sino el tipo de proceso así como la optimización de los parámetros implicados (Paredes y Solar, 2007)

En el presente estudio, el proceso productivo empleado para la producción de extracto de saponina a partir de Cucumis dipsaceus se inició con el macerado de pulpa de jaboncillo del campo con etanol de $70^{\circ} \mathrm{GL}$, filtrado, destilado para recuperar el alcohol, desengrasado y finalmente secado por atomización ; se asemeja al proceso especificado por Paredes y Solar (2007), donde especifica el proceso productivo de extracto iniciando con el macerado de la pulpa de jaboncillo del campo durante tres días empleando etanol como elemento extractante, seguido de un destilado a temperaturas entre $80^{\circ} \mathrm{C}$ a $85^{\circ} \mathrm{C}$ diferenciándose solamente que la destilación lo realizaron en tres etapas para eliminar el alcohol y finalmente proceder al secado por atomización obteniendo el extracto pulverizado seco como también lo reportaron Lee et al.,2018.

Al terminar la destilación se obtuvo un producto más concentrado en alcohol, esto representó un $90^{\circ} \mathrm{GL}$ en comparación con la concentración inicial, por lo tanto, el sistema de destilación continua en torre empacada con equivalente a 12 elementos de transferencia hace que sea más eficiente dicho proceso logrando una mejor rectificación del mismo (Lee et al.,2018). 
La eliminación de la grasa se efectuó en la misma columna de destilación teniendo en cuenta que constituye una extracción solido-liquida empleando como solvente etanol a una temperatura de $78^{\circ}$, permaneciendo la fase solida (pulpa de jaboncillo del campo) estacionaria en el reboyler de la columna de destilación. Giraldo (2010) evaluó diferentes solventes obteniendo una máxima extracción empleando hexano con una eficiencia de aproximadamente de $42 \%$ y con etanol obtuvo una extracción de 32\% de eficiencia extractiva (Giraldo, 2010); mientras que empleando la destilación continua para recuperar el etanol se logró separar un $23 \%$ de la grasa contenida en la pulpa de Cucumis dipsaceus, la cual se obtiene antes de obtener el destilado recuperado. Cabe resaltar como en todo proceso de producción se debe determinar el balance de materia, en la tabla 5; se efectuó el balance de materia expresándose en función del producto (extracto seco de saponina).

Tabla 5. Modelado del balance de materia en la producción de extracto de saponina de Cucumis dipsaceus.

\begin{tabular}{lcc}
\hline \multicolumn{1}{c}{ Corriente } & Modelo & Unidades \\
\hline Materia prima Cucumis dipsaceus & Mp $=5,20 \mathrm{Psec}$. & $\mathrm{kg} / \mathrm{h}$ \\
Disolvente (etanol) & Dis $=36,40 \mathrm{Psec}$. & $\mathrm{kg} / \mathrm{h}$ \\
Solución etanólica de saponina & Sol. $=41,60 \mathrm{Psec}$ & $\mathrm{kg} / \mathrm{h}$ \\
Solución etanólica de saponina filtrada & Solf. $=30,76 \mathrm{Psec}$. & $\mathrm{kg} / \mathrm{h}$ \\
Residuo sólido de Cucumis dipsaceus & Rsol $=10,84$ Psec. & $\mathrm{kg} / \mathrm{h}$ \\
Disolvente (etanol) recuperado & Disr. $=27,60 \mathrm{Psec}$. & $\mathrm{kg} / \mathrm{h}$ \\
Grasa extraída & Gras. $=1,20$ Psec. & $\mathrm{kg} / \mathrm{h}$ \\
Extracto de saponina & Ext. $=1,96$ Psec. & $\mathrm{kg} / \mathrm{h}$ \\
Producto (Extracto seco de saponina) & Psec. & $\mathrm{kg} / \mathrm{h}$ \\
\hline
\end{tabular}


Por otro lado, se realizó la determinación de los parámetros de diseño de los equipos implicados en el proceso de producción de extracto de saponinas de Cucumis dipsaceus. El molino de tipo uñas fue el equipo que se empleó en la fase de procesado (pretratamiento) de materia prima cuya función fue el pulpeado del fruto, iniciando con un algoritmo (modelado prescriptivo), como a continuación se presenta:

\section{Molino tipo uñas}

\section{Modelado para dimensionar molino}

INGRESAR: C: Materias prima a pulpear (Ton/h)

- Potencia mecánica del motor eléctrico: $\mathrm{P}=4,04 \mathrm{C}-0,5429$ (hp).

- Diámetro polea conducida: $\mathrm{DC}=0,0183 \mathrm{C}^{3}-0,1078 \mathrm{C}^{2}+0,2265 \mathrm{C}$ $+0,1102(\mathrm{~m})$

- Longitud del cilindro de rotación: $\mathrm{LC}=0,0699 \mathrm{C}^{3}-0,408 \mathrm{C}^{2}+0,8227 \mathrm{C}$ $+0,3575(\mathrm{~m})$

- Diámetro de la boca de carga: $\mathrm{DBC}=0,0183 \mathrm{C}^{3}-0,1078 \mathrm{C}^{2}+0,2265 \mathrm{C}$ $+0,1402(\mathrm{~m})$

- Diámetro polea matriz: $Ø \mathrm{mo}=0,0202 \mathrm{C}^{4}$ $-0,1726 \mathrm{C}^{3}+0,5238 \mathrm{C}-0,645 \mathrm{C}+0,3722(\mathrm{~m})$

- Diámetro polea conducida: $\varnothing \mathrm{C}=-0,001 \mathrm{C}^{2}+0,052 \mathrm{C}+0,2(\mathrm{~m})$

- Diámetro de la tolva : $\mathrm{DT}=0,0377 \mathrm{C}^{3}-0,2447 \mathrm{C}^{2}+0,5558 \mathrm{C}$ $+0,3817$ (m)

- Número de correas de perfil A y B: $\mathrm{NC}=-0,42 \mathrm{C}^{3}+1,94 \mathrm{C}^{2}-1,11 \mathrm{C}+1,64$

- IMPRIMIR: P; DC, LC; DBC, Ømo; ØC; DT; NC
Luego se procedió a proponer un modelado computacional (figura 2), con la finalidad de automatizar el proceso de pulpeado y a la vez obtener un programa computacional, el cual permite obtener los parámetros de diseño a una escala mayor (finalidad del scala up), según el dimensionamiento propuesto.

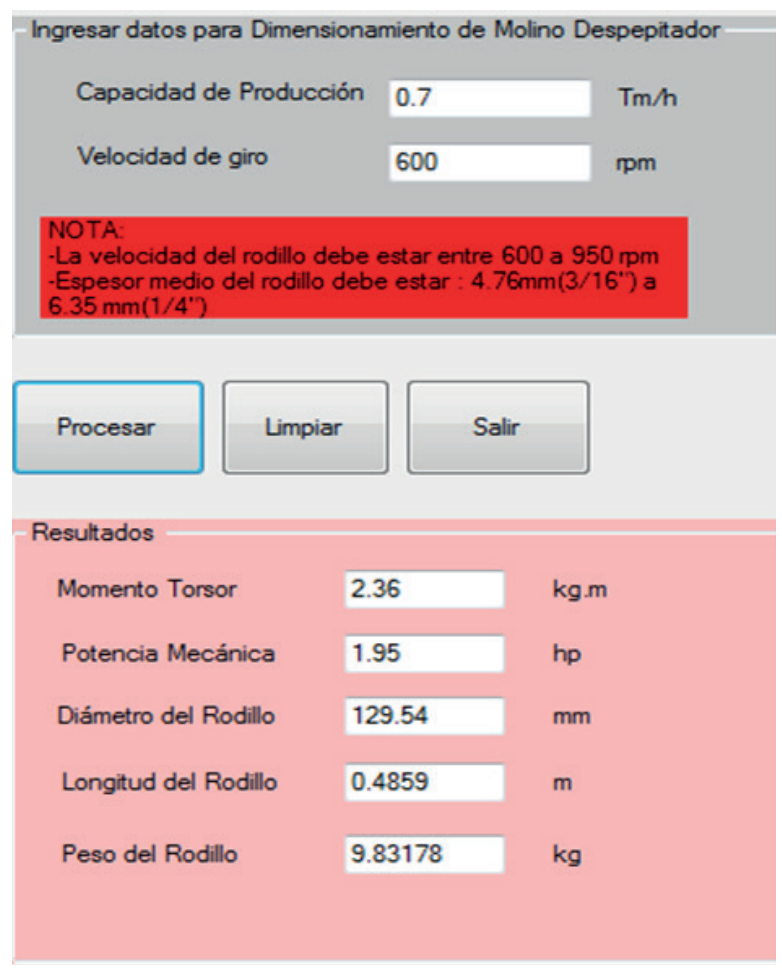

Figura 2. Molino tipo uñas para pulpeado del fruto Cucumis dipsaceus

El estudio y determinación de los parámetros de diseño también se realizaron para el equipo de la columna de destilación y sus componentes, como se detalla en la tabla 6 , estos parámetros se determinaron a través de pruebas experimentales a nivel piloto y escalado respectivo. 
Determinación de parámetros operativos para la producción de extracto de saponinas de Cucumis dipsaceus "jaboncillo de campo"

Tabla 6. Parámetros de diseño de los componentes de la columna de destilación

\begin{tabular}{lcc}
\hline \multicolumn{1}{c}{ Equipo } & Modelo & Unidades \\
\hline Superficie de transferencia del condensador & $\mathrm{A}=1,5 \mathrm{E}-5 \mathrm{Qc}$ & $\mathrm{m}^{2}$ \\
Flujo de agua de enfriamiento & $\mathrm{m}_{\mathrm{A}}=0,014 \mathrm{Qc}$ & $\mathrm{kg} / \mathrm{h}$ \\
Superficie de transferencia del reboyler & $\mathrm{S}=1,5 \mathrm{E}-6 \mathrm{Qr}$ & $\mathrm{m}^{2}$ \\
Flujo de agua de vapor saturado de la caldera & $\mathrm{W}=4,4 \mathrm{E}-4 \mathrm{Qr}$ & $\mathrm{kg} / \mathrm{h}$ \\
Diámetro de la columna de destilación tipo empacada & $\mathrm{dc}=0,0130 \mathrm{~F}^{0,5}$ & $\mathrm{~m}$ \\
Altura de la columna de destilación tipo empacada & $\mathrm{Hc}=0,1560 \mathrm{~F}^{0,5}$ & $\mathrm{~m}$ \\
\hline
\end{tabular}

Mientras que en la figura 3, se presenta un obtenido en base a los parámetros de diseño programa computacional en Visual Basic 10, que se obtuvieron (tabla 6).

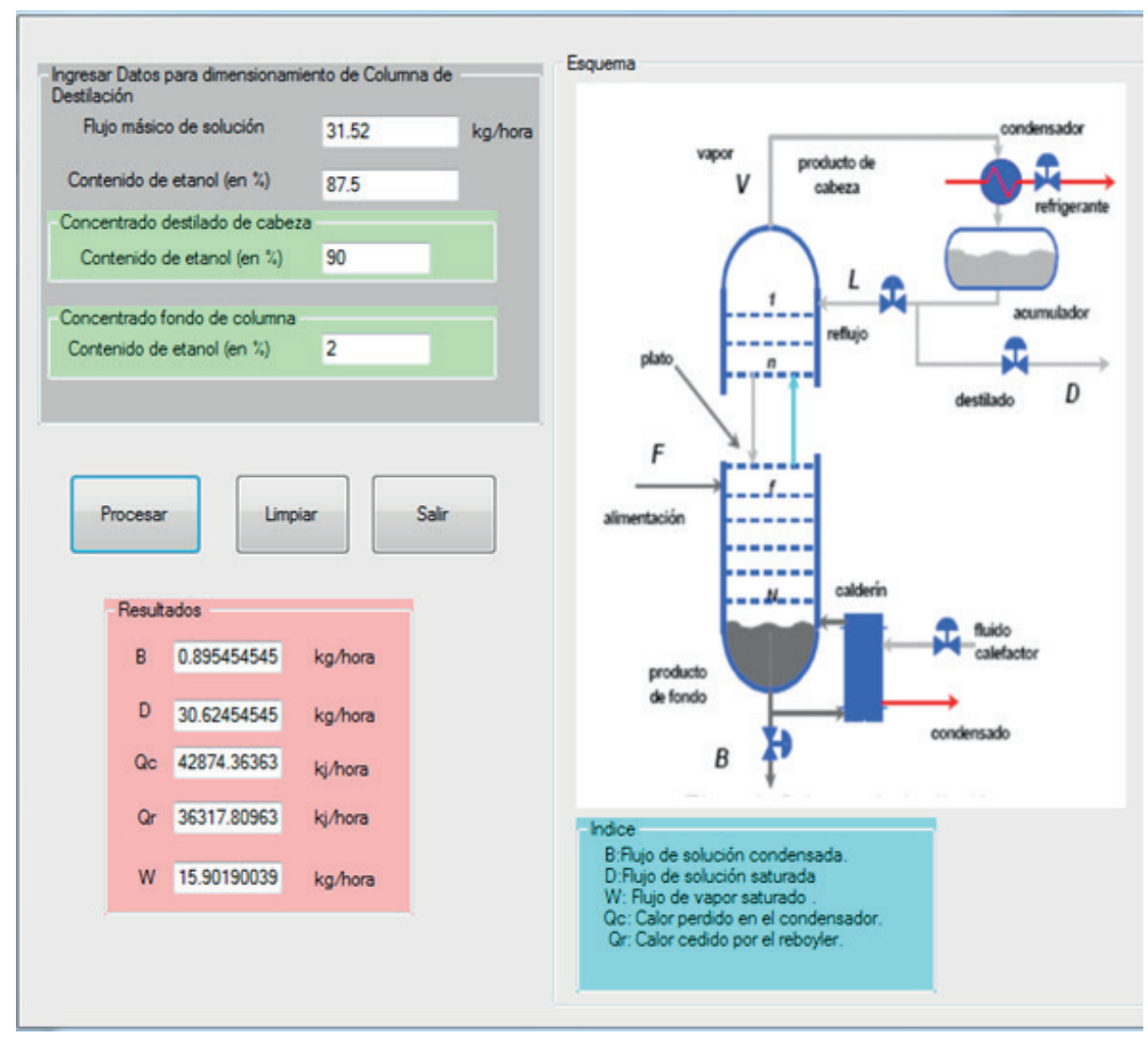

Figura 3. Balance de materia y energía en la columna de destilación tipo platos (Programa calculado mediante en Visual Basic10)

Por último, la etapa final en el proceso de producción de saponinas es el secado, considerando en la presente investigación el secado por atomización debido a que el producto se obtiene seco y particulado además el tiempo para la obtención del producto es corto (Lee et al.,2018)., por lo que también se empleó un equipo para la respectiva operación, para lo cual se obtuvo los parámetros de diseño y energéticos (tabla 7 y 8) así como la obtención del programa computacional (figura 4) obtenido a través del modelamiento realizado. 
Tabla 7. Parámetros de diseño de secador de tipo atomización para secado de extracto de saponina

\begin{tabular}{|c|c|c|}
\hline Especificación & Modelo & Unidades \\
\hline & Diámetro del secador & m \\
\hline \multirow{6}{*}{$\begin{array}{c}\text { Cuerpo del secador por } \\
\text { atomización }\end{array}$} & $0,2677732 \mathrm{~m}$ & \\
\hline & $D_{\mathrm{s}}=(0,0384 \text { Psec. })^{0,333}$ & \\
\hline & Altura del cilindro & m \\
\hline & $H_{C S}=(0,1283 \text { Psec. })^{0,333}$ & \\
\hline & Altura del Secador: & m \\
\hline & $\mathrm{H}_{\mathrm{S}}=(0,6912 \mathrm{Psec} .)^{0,333}$ & \\
\hline \multirow{2}{*}{$\begin{array}{c}\text { Bomba de dosificación de } \\
\text { alimentación }\end{array}$} & Potencia mecánica & \\
\hline & $P_{B}=0,92 P_{s e c} . /\left(1-H_{i}\right)$ & W \\
\hline \multirow{6}{*}{ Ventilador centrifugo } & Potencia & \multirow[b]{2}{*}{$(\mathrm{kW})$} \\
\hline & $P_{v}=0,1065 m_{p s}(k w)$ & \\
\hline & Diámetro del ciclón & \\
\hline & $D_{c}=0,1037$ Psec. ${ }^{0,333}$ & \\
\hline & Altura dela parte cilíndrica & \\
\hline & $\mathrm{H}=0,4148$ Psec. ${ }^{0,333}$ & \\
\hline \multirow[t]{11}{*}{ Ciclón } & Altura de la parte cónica & $\mathrm{m}$ \\
\hline & $h=0,1556$ Psec. $.0,333$ & \\
\hline & Boca de descarga del underflow & \\
\hline & $B=0,04 m_{p s}^{0,333}$ & \\
\hline & Boca de descarga del overflow & \\
\hline & $D_{e}=0,052 m_{p s}{ }^{0,333}$ & \\
\hline & Altura de la boca de entrada al ciclón & \\
\hline & $\mathrm{a}=0,052 \mathrm{~m}_{p s}, 0,333$ & \\
\hline & Anchura de la boca de entrada al & \\
\hline & ciclón & \\
\hline & $\mathrm{b}=0,0187 \mathrm{mps}_{\mathrm{ps}}{ }^{0,333}$ & \\
\hline
\end{tabular}

Tabla 8. Parámetros energéticos del secado de extracto seco de saponina

\begin{tabular}{ccc}
\hline Especificación & Modelo & Unidades \\
\hline Flujo másico de glp & $\mathrm{m}_{\text {glp }}=0,2595$ Psec. $+0,0018$ & $\mathrm{~kg} / \mathrm{h}$ \\
Flujo de calor requerido & Qsec. $=11856 \mathrm{Psec}$. & $\mathrm{kJ} / \mathrm{h}$ \\
Eficiencia térmica & 96,40 & $\%$
\end{tabular}




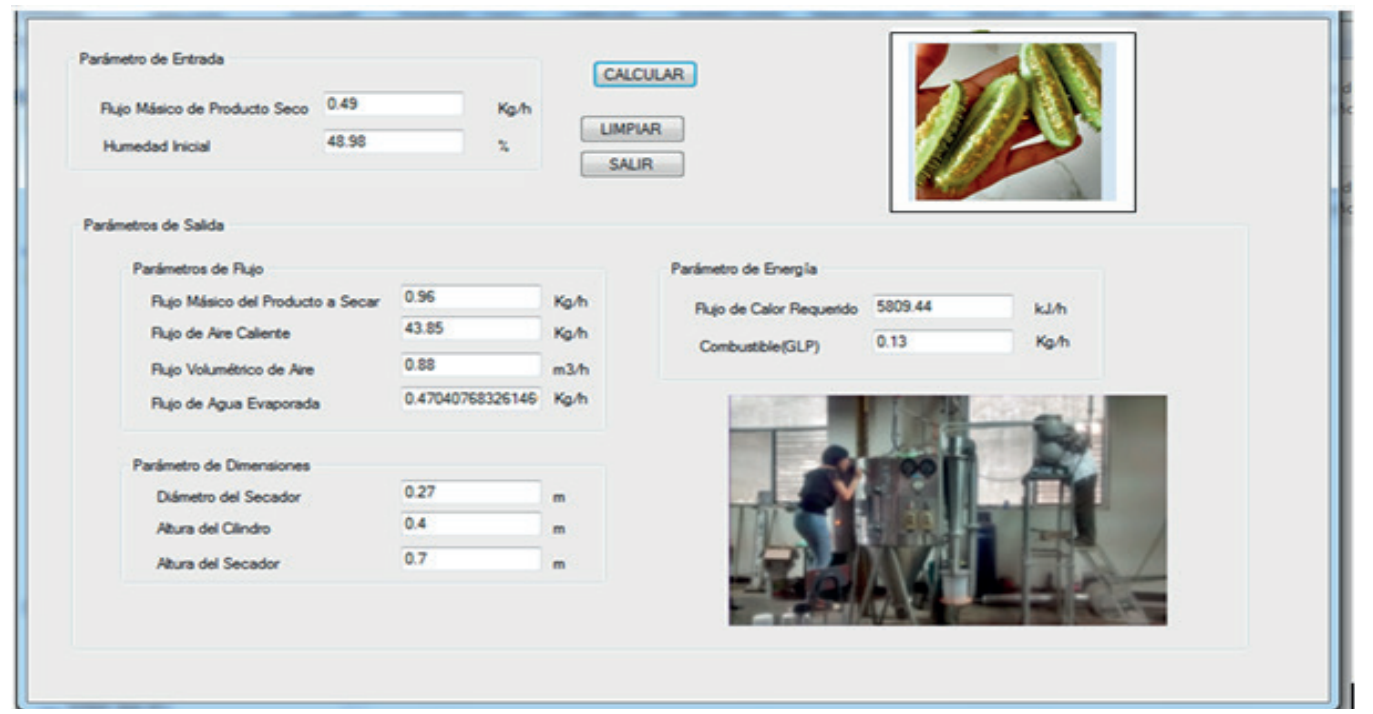

Figura 4. Programa computarizado para calcular parámetros de diseño y térmicos del secador de atomización.

\section{CONCLUSIONES}

Se obtuvo un rendimiento de $73,5 \%$ de saponinas de Cucumis dipsaceus en el proceso de extracción siendo los parámetros operativos: relación sólida/extractante de 1 , temperatura de $60^{\circ} \mathrm{C}$ y tiempo de extracción 30 minutos. Para realizar el escalamiento del proceso de producción de extracto de saponina de $\mathrm{Cu}$ cumis dipsaceus (jaboncillo de campo) primeramente se determinaron los parámetros operativos y a partir de ello se obtuvieron los respectivos balances de materia y energía, expresándolo en función de la capacidad de producción; elaborando los respectivos programas computarizados para obtener los parámetros de diseño de los equipos del proceso.

\section{REFERENCIAS BIBLIOGRÁFICAS}

Ahumada, A., Ortega, A., Chito, D y Benítez, R. (2016). Saponinas de quinua (Chenopodium quinoa Willd.): un subproducto con alto potencial biológico. Rev. Colomb. Cienc. Quím. Farm., 45(3), 438-469.

Bajad, P., Pardeshi, A y Pagore, V. (2019). Extraction, isolation and quantification of saponin from Dodonaea viscosa JACQ. The Phar- ma Innovation Journal 2019; 8(5): 41-44.

Bruneton, J. (2001). Saponósidos. In Farmacognosia, Fotoquímica, Plantas Medicinales. Zaragoza, España. Editorial Acribia, S.A. pp 664-709.

Castellanos, L., Fernández, A.O., Soto, R. y Martin, C. (2011). Efectividad Del Extracto De Furcraea hexapetala (Jacq.) Urban sobre Polyphagotarsonemus latus Banks en condiciones de laboratorio. Protección Vegetal, 26, (3): 12-18.

Flores, T. (2013). Estudio comparativo de tres metodologías cuantitativas de extracción de saponinas de la Melisa Officinalis "Toronjil". Per. Quim. Ing. Quìm., 16, 5.

Francis, G., Kerem, Z., Makkar,yH. Y Becker, K. (2002). The biological action of saponins in animal systems: A review. British J. Nutr. 88, 587-605.

Gianna, V. (2013). Extracción, Cuantificación y Purificación De Saponinas De Semillas De Chenopodium quinoa Willd provenientes del 
noroeste argentino. Tesis para obtener el grado de Doctor en Ciencias de la Ingeniería. Universidad Nacional de Córdoba Facultad de Ciencias Exactas, Físicas y Naturales.

Gianna, V., Montes, J.M., Calandri, E.L. y Guzmán, C.A. (2012). Impact of several variables on the microwave extraction of Chenopodium quinoa Willd saponins. International Journal of Food Science and Technology. 47, 1593-1597.

Giraldo, Y., Velásquez, J. y Cuartas, A. (2010). Extracción con solventes y purificación de aceite a partir de semillas de Jatropha curcas. Universidad Pontificia Bolivariana. Facultad de Ingeniería Química. B1. 11, Medellín, Colombia.

Güçlü-Üstündag, O. y Mazza, G. (2007). Saponins: Properties, applications and processing. Crit. Rev. Food Sci. Nutr. 47, 231-258.

Hernández, A. y Hermosilla, V. (2014). Efecto de la concentración de saponinas en la actividad hemolítica de extractos de ocho plantas de uso medicinal en Guatemala. Tesis para optar el título de Química bióloga. Facultad de ciencias químicas y farmacia. Universidad de San Carlos. Guatemala.

Hernández, Y. (2006). Estudio de la actividad hemolítica de los posibles taninos extraidos a partir de la Boldoa purpurascens Cav. Revista Electrónica de Veterinaria,2(10), 1-5.

Lee, A., Parks, S., Nguyen, M. y Roach, P. (2018). Effect of Solvents and Extraction Methods on Recovery of Bioactive Compounds from Defatted Gac (Momordica cochinchinensis Spreng.) Seeds. Separations 5(39):2-13. Lolli, S., Ying-Chieh, C., Sheng-Hsiang, W. y Gemine, V. (2020). Impact of meteorological conditions and air pollution on COVID-19 pandemic transmission in Italy. Scientific Reports 10:16213

Mostacero, L., Mejía, F. y Gamarra, O. (2002). Taxonomía de las fanerógamas útiles del Perú. CONCYTEC. Volumen I. Editorial Normas Legales.Trujillo.

Paredes, H. y Solar, M. (2007). Identificación y cuantificación de las saponinas contenidas en el fruto de la especie Cucumis dipsaceus por cromatografía de líquidos de alta resolución (HPLC). Tesis para optar el título de Químico farmacéutico. Facultad de Farmacia y Bioquímica. Universidad Nacional de Trujillo.

Prado, R. (2018). Evaluación de técnicas de extracción de saponinas de la quinua (Chenopodium quinoa willd) como alternativa de mejoramiento para la cadena productiva en Cundinamarca. Tesis para optar al título de: Especialización en procesos de alimentos y biomateriales. Universidad Nacional Abierta y a Distancia. UNAD.

Rijai,L. (2019). Review of Fast Technics for Isolation and Structure Elucidation of Triterpene Saponins Compound from Bionatural Products. J. Pharm. Sci. \& Res. 9(2), 2017, 150-158.

Villacrés, M., Calderón, C., Cauja, L., y Arcos, T. (2019). Obtención de detergente líquido usando saponina de quinua (Chenopodium quinoa Willd), chocho (Lupinus mutabilis Sweet) cabuya (Sisalana perrine) y su diseño de producción. Perfiles 21 (1):37-43. 